\title{
Application of Soft-Set Relations and Soft Matrices in Medical Diagnosis using Sanchez's Approach
}

\author{
Muhammad Naveed \\ Jafar \\ Department of \\ Mathematics \\ Lahore Garrison \\ University
}

Lahore - 54000, Pakistan

\author{
Muhammad Saqlain \\ Department of \\ Mathematics \\ Lahore Garrison \\ University, \\ Lahore-54000, Pakistan
}

\author{
Muhammad Saeed \\ Department of \\ Mathematics \\ University of \\ Management and \\ Technology \\ Lahore, Pakistan
}

\author{
Qamar Abbas \\ Department of Biology \\ Lahore Garrison \\ University \\ Lahore - 54000, Pakistan
}

\begin{abstract}
This article presents the concepts of soft set theory and its relations. It the common observation that more symptoms give more accuracy to calculate the disease, so we enhanced the work (Jafar at el., 2019) from trapezoidal to octagonal symptoms symbolically. So, we have used Sanchez's Approach in terms of Octagonal fuzzy number. In the end for the better understanding the theory an elaborative example using hypothetical data has also been presented.
\end{abstract}

\section{Keywords}

Fuzzy Number (FN), Octagonal Number, Soft Set (SS), Soft Matrix (SM) Sanchez's Method

\section{INTRODUCTION}

Provisional record is involved in most of the fields like engineering, economic and social disciplines etc. All such issues that a person encounters in his life cannot be solved by using ancient mathematical skills. In ancient arithmetic, digital model of a substance is formed and idea of specific classification of this traditional pattern is sought out. That's why, the digital data is much stunning, and the specific results can't come out.

There is various popular supposition to show errors in measurements. For example, (Zadeh, 1965) started Fuzzy set theory (FST), (Pawlak, 1999) Started rough set of theory. For very comprehensive concept regarding decision making Moldstove (1999) gave the concept of Soft set theory. Now a day's work on soft set theory make progress very quickly. In order to get over these difficulties, (Maji, Biswas and Roy, 2001) introduced the idea of Fuzzy soft set (FSS) like other computational procedures for illustration of obscurities which excluded all the strategies affecting the recent challenges. The theory of SS has powerful ability for adequate functions in various fields some of them were explain by many investigators. Recently, struggles on SS theory are getting famous really. Soft sets and Fuzzy softs sets are being used in decision making concepts of every field of life. (Saeed, M. 2018) has used TOPSIS in medical for diabetes investigations and (Saqlain et. al., 2019) introduced the TOPSIS by using accuracy function in his work and an application of MCDM is proposed. Application of fuzzy numbers in mobile selection in metros like Lahore is proposed by (Saqlain. M. et. al., 2019). TOPSIS technique of MCDM can also be used for the prediction of games, and it's applied in FIFA 2018 by (Saqlain. M. et. al. 2019). Prediction of games is a very complex topic and this game is also predicted by (Saqlain. M. et. al. 2019) Decision making is a very big problems for such a complex world. (Sanchez, 1976) gave an approach for decision making regarding medical diagnosis. (Jafar et.al, 2019) used Soft set theory and Sanchez approach in medical diagnosis using Trapezoidal numbers. In this study we are going to use the similar concept of diagnosis using Octagonal numbers.

\section{PRELIMINARIE}

\subsection{Soft Set [9]}

Let $\mathrm{U}$ be a discourse set and $\mathrm{S}$ be the set of parameters. The collection of power set of $U$ is denoted by $P(U)$, Then the soft set is denoted by a pair $(\mathrm{F}, \mathrm{A})$ and defined by function $\mathrm{F}: \mathrm{A} \rightarrow \mathrm{P}(\mathrm{U})$ where Áis the sub collection of the set of parameters.

Example: Let $U=\left\{m_{1}, m_{2}, m_{3}, m_{4}, m_{5}\right\}$ be a set of five mobile phones and $S=\left\{\mathrm{e}_{1}, \mathrm{e}_{2}, \mathrm{e}_{3}, \mathrm{e}_{4}\right\}$ be the set of four parameters which are 'expensive, beautiful, compatible, brand'. Let $\mathrm{A}$ be the sub collection of $\mathrm{S}$ which $\mathrm{A}=$ $\left\{\mathrm{e}_{2}, \mathrm{e}_{3}, \mathrm{e}_{4}\right\}$ so the soft set is $(\mathrm{F}, \hat{\mathrm{A}})=\left\{\left(\mathrm{e}_{2}, \mathrm{~F}\left(\mathrm{e}_{2}\right)\right),\left(\mathrm{e}_{3}, \mathrm{~F}\left(\mathrm{e}_{1}\right)\right),\left(\mathrm{e}_{4}, \mathrm{~F}\left(\mathrm{e}_{4}\right)\right)\right\}$ where

\begin{tabular}{lll}
\hline $\mathbf{F}\left(\mathbf{e}_{2}\right)$ & $\mathbf{F}\left(\mathbf{e}_{3}\right)$ & $\mathbf{F}\left(\mathbf{e}_{4}\right)$ \\
$=\left\{\mathrm{m}_{1}, \mathrm{~m}_{2}, \mathrm{~m}_{3}\right\}$ & $=\left\{\mathrm{m}_{3}, \mathrm{~m}_{5}\right\}$ & $=\left\{\mathrm{m}_{1}, \mathrm{~m}_{3}, \mathrm{~m}_{3}, \mathrm{~m}_{5}\right\}$
\end{tabular}

\subsection{Soft Relation [7-9]}

Consider two soft sets $(F, A)$ and $(G, B)$ over the same discourse set $U$, then the soft relation relation $(R, C)$ or simply $R$ in between soft sets $(F, A)$ and $(G, B)$ is the soft subset of $(\mathrm{F}, \mathrm{A}) \times(\mathrm{G}, \mathrm{B})$ where $\mathrm{C} \subseteq \mathrm{A} \times \mathrm{B}$ and for all $(\mathrm{a}, \mathrm{b}) \in \mathrm{C}$, $\mathrm{R}(\mathrm{a}, \mathrm{b})=\mathrm{H}(\mathrm{a}, \mathrm{b})$ where $(\mathrm{H}, \mathrm{A} \times \mathrm{B})=(\mathrm{F}, \mathrm{A}) \times(\mathrm{G}, \mathrm{B})$

\section{ALGORITHM}

a. Construct the soft sets of in patient's and symptoms.

b. Construct the soft set in symptoms and disease.

c. Write the matrix form of both 1 and 2 .

d. Compute the product matrix using given in methodology.

e. Using Mean all the values.

f. Make decision against highest value involved in the row. 


\section{METHODOLOGY}

\section{Sanchez's approach to disease identification using octagonal fuzzy number}

Let the set of patients $P=\left\{p_{1}, p_{2}, p_{3} \ldots . p_{n}\right\}$ with a set of symptoms $\mathrm{S}=\left\{\dot{\mathrm{s}}_{1}, \dot{\mathrm{s}}_{2}, \dot{s}_{3}, \ldots \dot{s}_{\mathrm{n}}\right\}$ and suppose the set of associated diseases $D=\left\{d_{1}, d_{2}, d_{3} \ldots d_{m}\right\}$

We employ FS notion to create strategy through Sanchez to analyse which patient is suffering from what disease. For this we build a Soft $S(F, S)$ over M where $G$ is a function F: P $\rightarrow$ $\mathrm{F}(\mathrm{s})$. This FSS offers the patient disease the patient disease sign matrix which is ground that delineate relation between patient and symptoms and is symbolised as T, where the entry items are FNs $\bar{s}$ constrict restrained by a octuplet $[(\mathrm{n}-$ 4), $(n-3),(n-2),(n-1),(n+1),(n+2),(n+3),(n+$ 4)].

At that point develop alternative FSS $(\mathrm{H}, \mathrm{M})$ upon A where $\mathrm{H}$ is a mapping $\mathrm{H}: \mathrm{S} \rightarrow \mathrm{F}(\mathrm{d})$. This FSS provide a relation matrix every component indicates the significance of the symptoms for a particular sickness. These components are considered identical to triangular FNs.

Hence the conventional form of $\mathrm{T}$ is

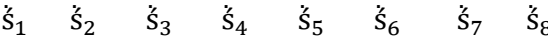
$\mathrm{p}_{1}\left[\begin{array}{llllllll}\tilde{\mathrm{a}}_{11} & \tilde{\mathrm{a}}_{12} & \tilde{\mathrm{a}}_{13} & \tilde{\mathrm{a}}_{14} & \tilde{\mathrm{a}}_{15} & \tilde{\mathrm{a}}_{16} & \tilde{\mathrm{a}}_{17} & \tilde{\mathrm{a}}_{18}\end{array}\right]$

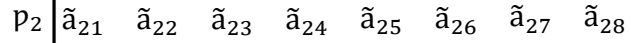
$p_{3}\left[\begin{array}{llllllll}\tilde{a}_{31} & \tilde{a}_{32} & \tilde{a}_{33} & \tilde{a}_{34} & \tilde{a}_{35} & \tilde{a}_{36} & \tilde{a}_{37} & \tilde{a}_{38}\end{array}\right]$

As well as the conventional form of $\mathrm{U}$ is

$$
\begin{aligned}
& \begin{array}{lll}
d_{1} & d_{2} & d_{3}
\end{array}
\end{aligned}
$$

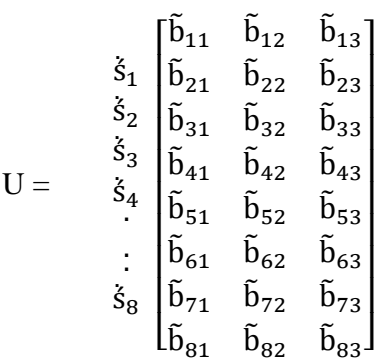

Presently executing the transformation operation $T \otimes U$, we procure the disease identification matrix of patient $\mathrm{D}^{*}$ like so,

$$
\begin{aligned}
& \begin{array}{lll}
d_{1} & d_{2} & d_{3}
\end{array} \\
& \mathrm{D}^{*}=\begin{array}{l}
\mathrm{p}_{1} \\
\mathrm{p}_{2} \\
\mathrm{p}_{3}
\end{array}\left[\begin{array}{lll}
\tilde{\mathrm{c}}_{11} & \tilde{\mathrm{c}}_{12} & \tilde{\mathrm{c}}_{13} \\
\tilde{\mathrm{c}}_{21} & \tilde{\mathrm{c}}_{22} & \tilde{\mathrm{c}}_{23} \\
\tilde{\mathrm{c}}_{31} & \tilde{\mathrm{c}}_{32} & \tilde{\mathrm{c}}_{33}
\end{array}\right] \\
& \text { Where } \mathrm{C}_{\mathrm{ij}} \text { is } \\
& C_{i j}=\left(\begin{array}{c}
\sum_{\mathrm{i}, \mathrm{j}=1}^{8}\left(\mathrm{a}_{\mathrm{ij}}-4\right)\left(\mathrm{b}_{\mathrm{ij}}-4\right) \\
\sum_{\mathrm{i}, \mathrm{j}=1}^{8}\left(\mathrm{a}_{\mathrm{ij}}-3\right)\left(\mathrm{b}_{\mathrm{ij}}-3\right) \\
\sum_{\mathrm{i}, \mathrm{j}=1}^{8}\left(\mathrm{a}_{\mathrm{ij}}-2\right)\left(\mathrm{b}_{\mathrm{ij}}-2\right) \\
\sum_{\mathrm{i}, \mathrm{j}=1}^{8}\left(\mathrm{a}_{\mathrm{ij}}-1\right)\left(\mathrm{b}_{\mathrm{ij}}-1\right) \\
\sum_{\mathrm{i}, \mathrm{j}=1}^{8}\left(\mathrm{a}_{\mathrm{ij}}+1\right)\left(\mathrm{b}_{\mathrm{ij}}+1\right) \\
\sum_{\mathrm{i}, \mathrm{j}=1}^{8}\left(\mathrm{a}_{\mathrm{ij}}+2\right)\left(\mathrm{b}_{\mathrm{ij}}+2\right) \\
\sum_{\mathrm{i}, \mathrm{j}=1}^{8}\left(\mathrm{a}_{\mathrm{ij}}+3\right)\left(\mathrm{b}_{\mathrm{ij}}+3\right) \\
\sum_{\mathrm{i}, \mathrm{j}=1}^{8}\left(\mathrm{a}_{\mathrm{ij}}+4\right)\left(\mathrm{b}_{\mathrm{ij}}+4\right)
\end{array}\right)
\end{aligned}
$$

All that point, averaging every component we get a brief $\begin{array}{llll}\text { disease identification matrix } D^{* *} & d_{1} & d_{2} & d_{3}\end{array}$

$$
\mathrm{D}^{* *}=\begin{aligned}
& \mathrm{p}_{1} \\
& \mathrm{p}_{2} \\
& \mathrm{p}_{3}
\end{aligned}\left[\begin{array}{lll}
\mathrm{v}_{11} & \mathrm{v}_{12} & \mathrm{v}_{13} \\
\mathrm{v}_{21} & \mathrm{v}_{22} & \mathrm{v}_{23} \\
\mathrm{v}_{31} & \mathrm{v}_{32} & \mathrm{v}_{33}
\end{array}\right]
$$

\section{CASE STUDY}

\section{Sanchez's approach to disease identification using octagonal fuzzy number}

Here we have 3 patients Bilal, Khurram and Salman in a hospital having disease signs fever, cerebral problem, and cough, loss of appetite, abdominal disorder, weight loss, headache and malaise. We consider a set $\mathrm{P}=\left\{\mathrm{p}_{1}, \mathrm{p}_{2}, \mathrm{p}_{3}\right\}$ as a

\begin{tabular}{|c|c|}
\hline $\boldsymbol{F}\left(\mathrm{p}_{1}\right)$ & 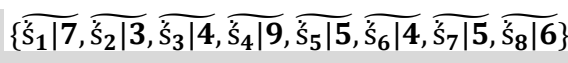 \\
\hline$F\left(\mathrm{p}_{2}\right)$ & 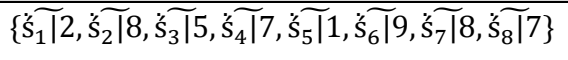 \\
\hline$F\left(p_{3}\right)$ & 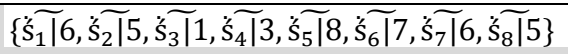 \\
\hline
\end{tabular}
ground set which symbolise the set of patients. $S=$ $\left\{\grave{s}_{1}, \grave{s}_{2}, \grave{s}_{3}, \ldots \grave{s}_{8}\right\}$ as a universal set for symptoms. And $\mathrm{D}=$ $\left\{d_{1}, d_{2}, d_{3}\right\}$ denotes the set for diseases as pyrexia, liver inflammation and pleurisy according to the disease signs.

We have

7 have 3 patients Bilal, khuram medical diagnosis.pezoida numbers. In this study we are going to use the similar concept of dThe FSS $(\mathrm{G}, \mathrm{S})$ is a parameterized group of all FS over M and offers an assemblage of a rough depiction of patientsdisease identification in the hospital. The FSS (G, S) describe the patient-disease sign matrix $\mathrm{T}$ (relation matrix)

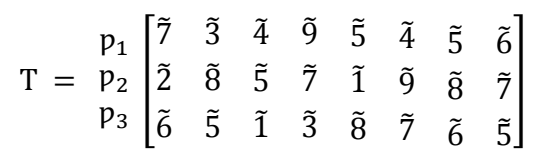

\begin{tabular}{|c|c|}
\hline$H\left(\dot{s}_{1}\right)$ & $\mathbf{H}\left(\dot{s}_{2}\right)$ \\
\hline$\left\{\overline{\mathrm{d}_{1} \mid 9}, \overline{\mathrm{d}_{2} \mid 5}, \overline{\mathrm{d}_{3} \mid 1}\right\}$ & $\left\{\overline{\mathrm{d}_{1} \mid 3}, \overline{\mathrm{d}_{2} \mid 5}, \overline{\mathrm{d}_{3} \mid 5}\right\}$ \\
\hline$H\left(\dot{s}_{5}\right)$ & $\mathrm{H}\left(\dot{s}_{6}\right)$ \\
\hline$\left\{\overline{\mathrm{d}_{1} \mid 1}, \overline{\mathrm{d}_{2} \mid 7}, \overline{\mathrm{d}_{3} \mid 3}\right\}$ & $\left\{\overline{\mathrm{d}_{1} \mid 6}, \overline{\mathrm{d}_{2} \mid 7}, \overline{\mathrm{d}_{3} \mid 6}\right\}$ \\
\hline$H\left(\dot{\mathrm{s}}_{3}\right)$ & $\mathrm{H}\left(\dot{\mathrm{s}}_{4}\right)$ \\
\hline$\left\{\overline{\mathrm{d}_{1} \mid 5}, \overline{\mathrm{d}_{2} \mid 2}, \overline{\mathrm{d}_{3} \mid 5}\right\}$ & $\mathrm{H}\left(\dot{\mathrm{s}}_{8}\right)$ \\
\hline$H\left(\dot{\mathrm{s}}_{7}\right)$ & $\left.\overline{\mathrm{d}_{2} \mid 8}, \overline{\mathrm{d}_{3} \mid 7}\right\}$ \\
\hline$\left\{\overline{\mathrm{d}_{1} \mid 8}, \overline{\mathrm{d}_{2} \mid 9}, \overline{\mathrm{d}_{3} \mid 5}\right\}$ & $\left\{\overline{\mathrm{d}_{1} \mid 5}, \overline{\mathrm{d}_{2} \mid 6}, \overline{\mathrm{d}_{3} \mid 9}\right\}$ \\
\hline
\end{tabular}

The FSS $(\mathrm{H}, \mathrm{M})$ is a parameterised group of all FS upon the set $M$ where $H: M \rightarrow G(D)$ and is established after special health credentials. Hence the FSS $(\mathrm{H}, \mathrm{M})$ offers an estimated delineation of the 3 disease and their symptoms. This SS is denoted by a relative matrix (symptom-disease matrix) $U$ and is presented via

$d_{1} \quad d_{2} d_{3}$ 


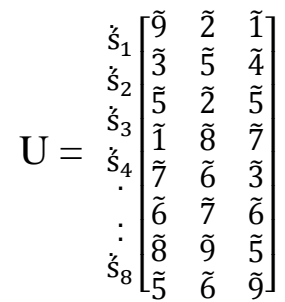

Presently executing the transformation operation $\mathrm{T} \otimes \mathrm{U}$, we acquire the disease identification matrix of patient $\mathrm{D}^{*}$ like so,

$$
\begin{aligned}
& \begin{array}{lll}
d_{1} & d_{2} & d_{3}
\end{array}
\end{aligned}
$$

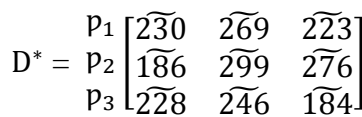

Where $\mathrm{C}_{\mathrm{ij}}$ is

$$
\mathrm{C}_{\mathrm{ij}}=\left(\begin{array}{c}
\sum_{\mathrm{i}, \mathrm{j}=1}^{8}\left(\mathrm{a}_{\mathrm{ij}}-4\right)\left(\mathrm{b}_{\mathrm{ij}}-4\right) \\
\sum_{\mathrm{i}, \mathrm{j}=1}^{8}\left(\mathrm{a}_{\mathrm{ij}}-3\right)\left(\mathrm{b}_{\mathrm{ij}}-3\right) \\
\sum_{\mathrm{i}, \mathrm{j}=1}^{8}\left(\mathrm{a}_{\mathrm{ij}}-2\right)\left(\mathrm{b}_{\mathrm{ij}}-2\right) \\
\sum_{\mathrm{i}, \mathrm{j}=1}^{8}\left(\mathrm{a}_{\mathrm{ij}}-1\right)\left(\mathrm{b}_{\mathrm{ij}}-1\right) \\
\sum_{\mathrm{i}, \mathrm{j}=1}^{8}\left(\mathrm{a}_{\mathrm{ij}}+1\right)\left(\mathrm{b}_{\mathrm{ij}}+1\right) \\
\sum_{\mathrm{i}, \mathrm{j}=1}^{8}\left(\mathrm{a}_{\mathrm{ij}}+2\right)\left(\mathrm{b}_{\mathrm{ij}}+2\right) \\
\sum_{\mathrm{i}, \mathrm{j}=1}^{8}\left(\mathrm{a}_{\mathrm{ij}}+3\right)\left(\mathrm{b}_{\mathrm{ij}}+3\right) \\
\sum_{\mathrm{i}, \mathrm{j}=1}^{8}\left(\mathrm{a}_{\mathrm{ij}}+4\right)\left(\mathrm{b}_{\mathrm{ij}}+4\right)
\end{array}\right)
$$

$$
\begin{aligned}
& C_{11}=\left\{\left(a_{11}-4\right)\left(b_{11}-4\right)+\left(a_{12}-4\right)\left(b_{21}-4\right)+\left(a_{13}-4\right)\left(b_{31}-4\right)+\left(a_{14}-4\right)\left(b_{41}-4\right)+\quad\left(a_{15}-4\right)\left(b_{51}-4\right)+\right. \\
& \left.\left(a_{16}-4\right)\left(b_{61}-4\right)+\left(a_{17}-4\right)\left(b_{71}-4\right)+\left(a_{18}-4\right)\left(b_{81}-4\right)\right\} \text {, } \\
& \left\{\left(a_{11}-3\right)\left(b_{11}-3\right)+\left(a_{12}-3\right)\left(b_{21}-3\right)+\left(a_{13}-3\right)\left(b_{31}-3\right)+\left(a_{14}-3\right)\left(b_{41}-3\right)+\quad\left(a_{15}-3\right)\left(b_{51}-3\right)\right. \\
& \left.+\left(a_{16}-3\right)\left(b_{61}-3\right)+\left(a_{17}-3\right)\left(b_{71}-3\right)+\left(a_{18}-3\right)\left(b_{81}-3\right)\right\} \text {, } \\
& \left\{\left(a_{11}-2\right)\left(b_{11}-2\right)+\left(a_{12}-2\right)\left(b_{21}-2\right)+\left(a_{13}-2\right)\left(b_{31}-2\right)+\left(a_{14}-2\right)\left(b_{41}-2\right)+\quad\left(a_{15}-2\right)\left(b_{51}-2\right)\right. \\
& \left.+\left(a_{16}-2\right)\left(b_{61}-2\right)+\left(a_{17}-2\right)\left(b_{71}-2\right)+\left(a_{18}-2\right)\left(b_{81}-2\right)\right\} \\
& \left\{\left(a_{11}-1\right)\left(b_{11}-1\right)+\left(a_{12}-1\right)\left(b_{21}-1\right)+\left(a_{13}-1\right)\left(b_{31}-1\right)+\left(a_{14}-1\right)\left(b_{41}-1\right)+\quad\left(a_{15}-1\right)\left(b_{51}-1\right)\right. \\
& \left.+\left(a_{16}-1\right)\left(b_{61}-1\right)+\left(a_{17}-1\right)\left(b_{71}-1\right)+\left(a_{18}-1\right)\left(b_{81}-1\right)\right\} \\
& \left\{\left(a_{11}+1\right)\left(b_{11}+1\right)+\left(a_{12}+1\right)\left(b_{21}+1\right)+\left(a_{13}+1\right)\left(b_{31}+1\right)+\left(a_{14}+1\right)\left(b_{41}+1\right)+\quad\left(a_{15}+1\right)\left(b_{51}+1\right)\right. \\
& \left.+\left(a_{16}+1\right)\left(b_{61}+1\right)+\left(a_{17}+1\right)\left(b_{71}+1\right)+\left(a_{18}+1\right)\left(b_{81}+1\right)\right\} \\
& \left\{\left(a_{11}+2\right)\left(b_{11}+2\right)+\left(a_{12}+2\right)\left(b_{21}+2\right)+\left(a_{13}+2\right)\left(b_{31}+2\right)+\left(a_{14}+2\right)\left(b_{41}+2\right)+\quad\left(a_{15}+2\right)\left(b_{51}+2\right)\right. \\
& \left.+\left(a_{16}+2\right)\left(b_{61}+2\right)+\left(a_{17}+2\right)\left(b_{71}+2\right)+\left(a_{18}+2\right)\left(b_{81}+2\right)\right\} \text {, } \\
& \left(a_{15}+3\right)\left(b_{51}+3\right) \\
& \begin{array}{r}
\left\{\left(a_{11}+3\right)\left(b_{11}+3\right)+\left(a_{12}+3\right)\left(b_{21}+3\right)+\left(a_{13}+3\right)\left(b_{31}+3\right)+\left(a_{14}+3\right)\left(b_{41}+3\right)\right. \\
\left.+\left(a_{16}+3\right)\left(b_{61}+3\right)+\left(a_{17}+3\right)\left(b_{71}+3\right)+\left(a_{18}+3\right)\left(b_{81}+3\right)\right\}
\end{array} \\
& \left\{\left(a_{11}+4\right)\left(b_{11}+4\right)+\left(a_{12}+4\right)\left(b_{21}+4\right)+\left(a_{13}+4\right)\left(b_{31}+4\right)+\left(a_{14}+4\right)\left(b_{41}-4\right)+\quad\left(a_{15}+4\right)\left(b_{51}+4\right)\right. \\
& \left.+\left(a_{16}+4\right)\left(b_{61}+4\right)+\left(a_{17}+4\right)\left(b_{71}+4\right)+\left(a_{18}+4\right)\left(b_{81}+4\right)\right\}
\end{aligned}
$$

$=(\{(7-4)(9-4)+(3-4)(3-4)+(4-4)(5-4)+(9-4)(1-4)+(5-4)(7-4)+(4-4)(6-4)+(5-4)(8-4)+(6-4)(5-4)\}$,

$\{(7-3)(9-3)+(3-3)(3-3)+(4-3)(5-3)+(9-3)(1-3)+(5-3)(7-3)+(4-3)(6-3)+(5-3)(8-3)+(6-3)(5-3)\}$,

$\{(7-2)(9-2)+(3-2)(3-2)+(4-2)(5-2)+(9-2)(1-2)+(5-2)(7-2)+(4-2)(6-2)+(5-2)(8-2)+(6-2)(5-2)\}$,

$\{(7-1)(9-1)+(3-1)(3-1)+(4-1)(5-1)+(9-1)(1-1)+(5-1)(7-1)+(4-1)(6-1)+(5-1)(8-1)+(6-1)(5-1)\}$, $\{(7+1)(9+1)+(3+1)(3+1)+(4+1)(5+1)+(9+1)(1+1)+(5+1)(7+1)+(4+1)(6+1)+(5+1)(8+1)+(6+1)(5+1)\}$, $\{(7+2)(9+2)+(3+2)(3+2)+(4+2)(5+2)+(9+2)(1+2)+(5+2)(7+2)+(4+2)(6+2)+(5+2)(8+2)+(6+2)(5+2)\}$, $\{(7+3)(9+3)+(3+3)(3+3)+(4+3)(5+3)+(9+3)(1+3)+(5+3)(7+3)+(4+3)(6+3)+(5+3)(8+3)+(6+3)(5+3)\}$, $\{(7+4)(9+4)+(3+4)(3+4)+(4+4)(5+4)+(9+4)(1+4)+(5+4)(7+4)+(4+4)(6+4)+(5+4)(8+4)+(6+4)(5+4)\})$

$=(\{(3)(5)+(-1)(-1)+0+(5)(-3)+(1)(3)+0+(1)(4)+(2)(1)\}$,

$\{(4)(6)+0+(1)(2)+(6)(-2)+(2)(4)+(1)(3)+(2)(5)+(3)(2)\}$,

$\{(5)(7)+(1)(1)+(2)(3)+(7)(-1)+(3)(5)+(2)(4)+(3)(5)+(4)(3)\}$,

$\{(6)(8)+(2)(2)+(3)(4)+0+(4)(6)+(3)(5)+(4)(6)+(5)(4)\}$,

$\{(8)(10)+(4)(4)+(5)(6)+(10)(2)+(6)(8)+(5)(7)+(6)(9)+(7)(6)\}$,

$\{(9)(11)+(5)(5)+(6)(7)+(11)(3)+(7)(9)+(6)(8)+(7)(10)+(8)(7)\}$,

$\{(10)(12)+(6)(6)+(7)(8)+(12)(4)+(8)(10)+(7)(9)+(8)(11)+(9)(8)\}$,

$\{(11)(13)+(7)(7)+(8)(9)+(13)(5)+(9)(11)+(8)(10)+(9)(12)+(10)(9)\})$

$=\{15+1-15+3+4+2\},\{24+2-12+8+3+10+6\}$, 
$\{5+1+6-7+15+8+15+12\},\{48+4+12+24+15+24+20\},\{80+16+30+20+48+35+54+42\}$,

$\{99+25+42+33+63+48+70+56\},\{120+36+56+48+80+63+88+72\},\{143+49+72+65+99+80+108+90\})$

$\boldsymbol{C}_{\mathbf{1 1}}=(10,41,88,143,325,436,563,706)$

\begin{tabular}{|c|c|c|c|}
\hline $\begin{aligned} & C_{i j} \\
& \forall i \& j\end{aligned}$ & Order Octuplet & Average & Values \\
\hline$C_{11}$ & $(10,41,88,143,325,436,563,706)$ & $\frac{(10+41+88+143+325+436+563+706)}{8}$ & 289 \\
\hline$C_{12}$ & $(33,68,119,186,368,483,614,761)$ & $\frac{(33+68+119+186+368+483+614+761)}{8}$ & 329 \\
\hline$C_{13}$ & $(15,43,87,147,315,423,547,687)$ & $\frac{(15+43+87+147+315+423+547+687)}{8}$ & 283 \\
\hline$C_{21}$ & $(3,33,108,167,333,448,579,726)$ & $\frac{(3+33+108+167+333+448+579+726)}{8}$ & 299.6 \\
\hline$C_{22}$ & $(29,86,141,212,388,493,620,741)$ & $\frac{(29+86+141+212+388+493+620+741)}{8}$ & 338.7 \\
\hline$C_{23}$ & $(52,84,132,192,372,484,612,756)$ & $\frac{(52+84+132+192+372+484+612+756)}{8}$ & 335.5 \\
\hline$C_{31}$ & $(28,69,12,171,435,591,623,706)$ & $\frac{(28+69+12+171+435+591+623+706)}{8}$ & 329.3 \\
\hline$C_{32}$ & $(26,57,104,167,341,452,592,722)$ & $\frac{(26+57+104+167+341+452+592+722)}{8}$ & 307.6 \\
\hline$C_{33}$ & $(-8,16,56,128,272,376,496,632)$ & $\frac{(-8+16+56+128+272+376+496+632)}{8}$ & 246 \\
\hline
\end{tabular}

Hence after de averaging the exceeding matrix is

$D^{* *}=p_{1} p_{2}\left[\begin{array}{ccc}289 & 329 & 283 \\ 299.6 & 338.7 & 335.5 \\ 329.3 & 307.6 & 246\end{array}\right]$

It is observed by the above table the patient $p_{1}$ and $p_{2}$ are suffering from $d_{2}$ andp $p_{3}$ is experiencing $d_{1}$

\section{CONCLUSION}

In this study we enhanced the concept that the applications of soft matrices along with Sanchez's approach. If we increase number of symptoms, then it gives us more accuracy in disease identification. Results are compared with the results of (Jafar.et al., 2019) and proposed technique gives us more accuracy in results. Thus, in future Sanchez's approach along with soft matrices can be implemented to identify the diseases and more accuracy can be gained by increasing the number of symptoms.

\section{REFERENCES}

[1] Ali, Ml, Feng, F, Liu, X, Min, WK, Shabir, M (2009) On some new operations in soft set theory. Comput. Math. Appl. 57. 1547-1553

[2] Ali, Ml, Shabir, M (2010) Comments on De Morgan's law in fuzzy soft sets. J. Fuzzy Math. 18(3), 679-686.

[3] Chetia, B, Das, PK (2010) An application of interval valued fuzzy soft set in medical diagnosis. Int. J. Contemp. Math. Sci. 5(38), 1887-1894

[4] De, SK. Biswas, R, Roy, AR (2001) An application of intuitionistic fuzzy sets in medical diagnosis. Fuzzy sets syst. 117. 209-213

[5] Jafar.N.M,Muniba.K,Saeed.A,Abbas.S,Bibi.I.(2019), Application of Sanchez's Approach to Disease Identification Using Trapezoidal Fuzzy Numbers. International Journal of Latest Engineering Research and Applications.4(9):51-57 
[6] Kaur.A, Kumar,A (2011) A new method for solving fuzzy transportation problems using ranking function, Appl. Math. Model. 35:5652-5661.

[7] Maji, PK, Biswas, R. Roy, AR (2001) Fuzzy soft set. $J$. Fuzzy Math. 9(3): 677-692

[8] Meenakshi, AR, Kaliraja, M (2011) An application of interval valued fuzzy matrices in medical diagnosis. Int. J. Math. Anal. 5(36), 1791-1802

[9] Molodtsov, D (1999) Soft set theory first result. Comput. Math. Appi. 37 :19-31

[10] Neog, TI. Sut, DK (2011) Theory of fuzzy soft sets from a new perspective. Int. J Latest Trends Computers. 2(3): $439-450$

[11] Nieto-Morote,A, F. Ruz-Vila (2011) A fuzzy approach to construction project risk assessment, Int. J. Project Manage. 29: 220-231.

[12] Pawlak, Z (1999) Rough sets. Int. J. Comput. Inf. Sci. 11:341-356

[13] Saikia, BK, Das, Pk, Borkakati, AK (2003) An application of intuitionistic fuzzy soft sets in medical diagnosis. Bio-Sci. Res. Bull. 19(2):121-127

[14] Riaz. M., Saqlain. M. and Saeed. M. (2019). Application of Generalized Fuzzy TOPSIS in Decision Making for Neutrosophic Soft set to Predict the Champion of FIFA 2018: A Mathematical Analysis, Punjab University
Journal of Mathematics PUJM, 51(8): 111-126.

[15] Saeed, M., Zulqarnain, M. and Dayan, F. (2018). TOPSIS analysis for the prediction of diabetes based on general characteristics of humans. Int. J. of Pharm. Sci. and Research. 9. 2932-2939.

[16] Saqlain M, Saeed M, Ahmad M. R, Smarandache F, (2019), Generalization of TOPSIS for Neutrosophic Hypersoft set using Accuracy Function and its Application, Neutrosophic Sets and Systems (NSS), 27: 131-137.

[17] Saqlain. M., Jafar. N. and Riffat. A., (2019). Smart phone selection by consumers' in Pakistan: FMCGDM fuzzy multiple criteria group decision making approach, Gomal University Journal of Research, GUJR, 34(1): 27-31.

[18] Saqlain M, Jafar N, Hamid R, Shahzad A. (2019). Prediction of Cricket World Cup 2019 by TOPSIS Technique of MCDM-A Mathematical Analysis, International Journal of Scientific \& Engineering Research, IJSER, 10(2): 789-792.

[19] Sanchez, E (1976) Resolution of composite fuzzy relation equations. Inf. Control 30, 38-48

[20] Sanchez, E (1979) Inverse of fuzzy relations, application to possibility distributions and medical diagnosis. Fuzzy Sets Syst. 2(1):75-86

[21] Zadeh. L.A. (1965) Fuzzy sets. Information control 8: 338-348 